\section{Waist circumference percentile curves as a screening tool to predict cardiovascular risk factors and metabolic syndrome risk in Brazilian children}

\author{
Curvas de percentis do perímetro de cintura \\ como ferramenta de rastreamento para predi- \\ zer fatores de risco cardiovasculares e risco de \\ síndrome metabólica em crianças brasileiras
}

\section{Curvas de percentil de circunferencia de cintura como herramienta de detección para predecir factores de riesgo cardiovascular y síndrome metabólico en niños brasileños}

\author{
Alynne Christian Ribeiro Andaki 1 \\ Edmar Lacerda Mendes 1 \\ Amanda Santos 2 \\ Ciro Jose Brito 3 \\ Adelson Luiz Araújo Tinôco 4 \\ Jorge Mota 2
}

doi: 10.1590/0102-311X00105317

\begin{abstract}
The goals of this study were to develop reference values for waist circumference (WC) in Brazilian children between 6-10 years old and to evaluate the $W C$ performance in predicting cardiovascular risk factors and metabolic syndrome (MetS) in children. This is a population-based epidemiological crosssectional study, in which 1,397 children participated, with a 6-10 years old probability sampling and from public and private schools in the city of Uberaba, Minas Gerais State, Brazil. WC was measured at the waist narrowest point (WC1) and at the umbilicus level (WC2). Blood samples and blood pressure were collected to determine the MetS diagnosis. There was a significant effect of age $(p=0.001)$, anatomical point (WC1 vs. WC2, $p=0.001$ ) and sex-anatomical point interaction $(p=0.016)$ for WC. Smoothed sex-and agespecific 5th, 10th, 25th, 50th, 75th, 90th and 95th percentile curves of WC1 and WC2 were designed by the LMS method. WC was accurate to predict $M e t S$, for all ages [area under the ROC curve (AUC) $>0.79$ and $p<0.05$ ], regardless of sex. This study presented percentile curves for WC at two anatomical points in a representative sample of Brazilian children. Furthermore, WC was shown to be a strong predictor of cardiovascular risk factors and MetS in children.
\end{abstract}

Anthropometry; ROC Curve; Child Development

\author{
Correspondence \\ A. C. R. Andaki \\ Departamento de Ciências do Esporte, Universidade Federal do \\ Triângulo Mineiro. \\ Av. Getúlio Guarita 159, Uberaba, MG 38025-180, Brasil. \\ alynneandaki@yahoo.com.br

\footnotetext{
1 Departamento de Ciências do Esporte, Universidade Federal do Triângulo Mineiro, Uberaba, Brasil.

2 Centro de Investigação em Actividade Física, Saúde e Lazer, Universidade do Porto, Porto, Portugal.

3 Faculdade de Educação Física, Universidade Federal de Juiz de Fora, Juiz de Fora, Brasil.

4 Departamento de Nutrição e Saúde, Universidade Federal de Viçosa, Viçosa, Brasil.
} 


\section{Introduction}

Waist circumference (WC) is considered the most accurate anthropometric method to predict cardiovascular risk factors and metabolic syndrome (MetS) in children 1,2,3,4,5,6. Children with the highest WC values had the largest mean values of body fat mass, intima-media thickness, body mass index (BMI), the lowest values of cardiorespiratory fitness and moderate and vigorous physical activity intensity 7 . Moreover, an increased WC may decrease aerobic capacity in children ${ }^{8}$. Furthermore, WC was positively associated with higher household income and greater availability of electronic devices and motorized transport at home 9 .

However, body fat distribution is not routinely measured in clinical practice. Routine clinical practices must incorporate WC measurements, in addition to traditional measurements, given that normal and overweight children who are central obese may not be identified as such through traditional methods 10 .

Different countries developed reference curves for their population and chose different anatomical points for measurements. The adopted measurement for Canadian children and adolescents 11 , rural Portuguese children 12 and Japanese girls 13 on WC reference curves was the narrowest waist point, on the other, in Australian 14 and Japanese 15 children the measurement was performed at the umbilicus level.

Studies point the increase in the WC of children over the 20th century in many countries $16,17,18$, this measurement can be used as a reference method to predict MetS and other cardiovascular risk factors in children. However, few studies have attempted to validate cutoff points for reference curves as predictors of cardiovascular risk factors 19 and MetS 15. The development of these curves must be directly associated with the prediction of cardiovascular risk factors and MetS risk, as these children have already been affected in childhood. Therefore, the goal of this study is to develop reference values for WC in Brazilian children aged 6-10 year old and evaluate the WC performance in predicting cardiovascular risk factors and MetS risk in children.

\section{Methods}

\section{Study design}

This is a population-based epidemiological cross-sectional study with a 6-10 years old probability sampling, from public and private schools located in the urban and rural areas of the municipality of Uberaba, Brazil. Uberaba is located in the state of Minas Gerais, in the South-eastern region of Brazil with a population of 318,813 inhabitants and a Human Development Index (HDI) of 0.772.

\section{Sampling}

Sample calculation comprised the number of children enrolled in primary education ( 1 st to $9^{\text {th }}$ year of education), a prevalence of $50 \%$ central obesity (unknown prevalence in the municipality), $3.5 \%$ standard error and $95 \%$ confidence level. The minimum sample size was $n=768$ children and subsequent additional $10 \%$ to compensate for losses and refusals, and $20 \%$ to minimize confounding factors, totaling $\mathrm{n}=1,014$ children.

For sample selection, the schools were stratified according to the school classification (municipal, state and private). Fifteen eligible schools in Uberaba were randomly selected. The number of children from each stratum was determined in proportion to the number of enrollments, according to data provided by the Department of Education of the state.

\section{Procedures}

After approval by the Ethics Committee on Human Research of the Federal University of the Triângulo Mineiro (protocol n. 1710), the school principals were contacted to obtain authorization and schedule the data collection procedures. Students who met the inclusion criteria and were interested 
in participating in the study received an Informed Consent Form for acknowledgment and for their parents' signature. WC, blood samples and blood pressure were performed at school following specific protocols.

\section{Measurements}

Body mass was obtained by a digital electronic scale and the height $(\mathrm{H})$ was obtained using a portable stadiometer. BMI was calculated by dividing the body mass $(\mathrm{kg})$ by the squared height $\left(\mathrm{m}^{2}\right)$. Children were classified as overweight and obese according to the International Obesity Task Force (IOTF) criteria 20.

WC was measured after normal expiration using a flexible and non-elastic $2 \mathrm{~m}$ tape (Sanny, São Paulo, Brazil). All measurements were performed in triplicate and the average was recorded. The measurements were taken at the following anatomic sites: at the narrowest point of the waist, between the iliac crest and the ribs (WC1), as recommended by the Anthropometric Standardization Reference Manual 21, and at the umbilicus level (WC2).

Blood samples $(8 \mathrm{~mL})$ were collected in BD Vacutainer vacuum tubes and centrifuged at $3,400 \mathrm{rpm}$ for eight minutes to separate serum and plasma from other blood components. Serum samples were analyzed for the determination of high-density lipoprotein-cholesterol (HDL-c) and triglycerides, and plasma for blood glucose levels. A semi-automated Bio 200F analyzer (Bioplus, São Paulo, Brazil) was used to determine the blood variables, following the standards and technical specifications of the used reagents.

The following cardiovascular risk factors criteria were considered: hyperglycemia (fasting glucose $\geq 100 \mathrm{mg} / \mathrm{dL} 22$; hypertriglyceridemia $\geq 100 \mathrm{mg} / \mathrm{dL}$ ( $\leq 9$ years) and $\geq 130 \mathrm{mg} / \mathrm{dL}$ ( $>10$ years); HDL-c $<40 \mathrm{mg} / \mathrm{dL} 23$.

Blood pressure was measured using a sphygmomanometer according to standardized approaches 24. Only children whose systolic or diastolic blood pressure values exceeded the 90th percentile (according to height, sex and age) in three different measurements were diagnosed with high blood pressure 25 .

MetS diagnosis was determined by the presence of at least three of the following cardiovascular risk factors: obesity (BMI) 20, high blood pressure 25, hypertriglyceridemia 23, HDL-c below recommended values 23 and hyperglycemia 22 .

\section{Statistical analysis}

Univariate analysis of variance (ANOVA) was performed to test the main effects of age (6-10 years old), sex (male and female), anatomical point (WC1 and WC2) and interactions (age ${ }^{*}$ sex, age*anatomical point, sex ${ }^{*}$ anatomical point and age* sex $^{*}$ anatomical point). Statistical analyses were performed using the IBM SPSS software, version 24 for Windows (IBM Corp., Armonk, USA).

WC percentile curves were calculated with the LMS ChartMaker Pro software, version 2.54 (The Institute of Child Health, London. http://lmschartmaker.software.informer.com/2.5/) 26. The LMS method assumes that data can be normalized by using power transformation. Percentile curves were designed to smooth specific age and sex curves: $L$ for skewness, $M$ for median and $S$ for coefficient of variation. Further comparisons should hold $5^{\text {th }}, 10^{\text {th }}, 25^{\text {th }}$, 50th, $75^{\text {th }}, 90^{\text {th }}$ and $95^{\text {th }}$ percentiles as anatomical point. The sample for each sex and age groups was separated into 100 or more subjects, a number considered as the suitable minimum for the LMS method. In each layer, L, M and S parameters were calculated 27 .

Cutoff points for WC were suggested using receiver operating characteristic (ROC) curves. The optimal cutoffs were determined by finding the values that allowed the best balance between sensitivity and specificity. The statistical significance of each analysis was assessed by the area under the ROC curve (AUC) and by the inferior limit of the 95\% confidence interval (95\%CI) being $>0.528$. 


\section{Results}

The study included 1397 children of both sexes, mean age of $8.55 \pm 1.53$ years, and most of the children were girls (52.2\%). High blood pressure, hyperglycemia, HDL-c below recommended and hypertriglyceridemia were found in $11.3 \%, 0.9 \%, 43.1 \%, 12.2 \%$ children, respectively. The prevalence of overweight, obesity and MetS in the sample was 13.5\%, 5.0\% and 3.5\% respectively.

Descriptive statistics for WC by sex are shown in Table 1. A univariate ANOVA including WC as the dependent variable and age, sex and anatomical point as fixed factors showed significant main effects for age $\left[\mathrm{F}_{(4,2770)}=94.334, \mathrm{p}=0.001\right]$, anatomical point $\left[\mathrm{F}_{(1,2770)}=141.756, \mathrm{p}=0.001\right]$ and sex anatomical point interaction $\left[\mathrm{F}_{(1,2770)}=5.814, \mathrm{p}=0.016\right]$, but not for $\operatorname{sex}\left[\mathrm{F}_{(1,2770)}=0.125\right.$, $\mathrm{p}=0.724]$.

Smoothed sex- and age-specific percentiles for WC1 and WC2 are presented in Figure 1 and Table 2. In general, WC values increased with age in both sexes, regardless of the anatomical point.

WC showed high sensitivity in predicting metabolic syndrome, with values above the 50th percentile, however, most of the risk values are above the $75^{\text {th }}$ percentile (Table 3). The AUC for WC1 presented higher values than WC2. Furthermore, a specific cutoff point by age and sex for WC to predict CVRF in girls and boys was suggested (Table 4). Note that the percentile results are shown in approximate values to facilitate their use in clinical practice.

\section{Table 1}

Waist circumference (mean \pm standard deviation) in 6-10 years old children. Uberaba, Minas Gerais State, Brazil, 2011-2012.

\begin{tabular}{|c|c|c|c|c|c|c|}
\hline & \multicolumn{3}{|c|}{ Girls } & \multicolumn{3}{|c|}{ Boys } \\
\hline & n & WC1 & WC2 & $\mathbf{n}$ & WC1 & WC2 \\
\hline \multicolumn{7}{|c|}{ Age (years) } \\
\hline 6 & 116 & $54.3 \pm 5.6$ & $58.0 \pm 7.1$ & 118 & $55.1 \pm 5.35$ & $57.1 \pm 6.5$ \\
\hline 7 & 161 & $56.1 \pm 6.6$ & $59.9 \pm 8.3$ & 164 & $57.0 \pm 6.68$ & $59.1 \pm 7.9$ \\
\hline 8 & 177 & $58.3 \pm 6.6$ & $62.9 \pm 8.1$ & 134 & $58.8 \pm 7.10$ & $61.8 \pm 8.8$ \\
\hline 9 & 157 & $60.7 \pm 8.1$ & $65.5 \pm 9.8$ & 131 & $60.9 \pm 8.24$ & $64.5 \pm 9.8$ \\
\hline 10 & 118 & $61.5 \pm 9.0$ & $66.8 \pm 10.7$ & 121 & $63.4 \pm 8.98$ & $67.2 \pm 10.5$ \\
\hline Total & 729 & $58.2 \pm 7.6$ & $62.7 \pm 9.4$ & 668 & $59.0 \pm 7.85$ & $61.9 \pm 9.6$ \\
\hline \multicolumn{7}{|c|}{ ANOVA } \\
\hline & & & & \multicolumn{3}{|c|}{ p-value } \\
\hline \multicolumn{7}{|c|}{ Main effects } \\
\hline Age & & & & \multicolumn{3}{|c|}{0.001} \\
\hline Sex & & & & \multicolumn{3}{|c|}{0.724} \\
\hline Bench & and $W$ & & & \multicolumn{3}{|c|}{0.001} \\
\hline \multicolumn{7}{|c|}{ Interaction effects } \\
\hline $\operatorname{Age}^{*} \mathrm{k}$ & & & & \multicolumn{3}{|c|}{0.289} \\
\hline $\mathrm{Age}^{*} \mathrm{~S}$ & & & & \multicolumn{3}{|c|}{0.598} \\
\hline $\mathrm{Sex}^{\star} \mathrm{b}$ & & & & \multicolumn{3}{|c|}{0.016} \\
\hline $\mathrm{Age}^{*} \mathrm{~S}$ & & & & \multicolumn{3}{|c|}{0.993} \\
\hline
\end{tabular}

ANOVA: univariate analysis of variance; WC1: waist circumference measurement at the narrowest point;

WC2: waistcircumference measurement at the umbilicus level. 


\section{Figure 1}

Smoothed reference curves for the 5 th, 10 th $, 25^{\text {th }}, 50^{\text {th }}, 75^{\text {th }}$, 90th and $95^{\text {th }}$ percentiles of waist circumference measured (1a) at the narrowest point of the waist, between the iliac crest and the ribs (WC1) and (1b) at the umbilicus level (WC2) in Brazilian 6-10 years old boys and girls.
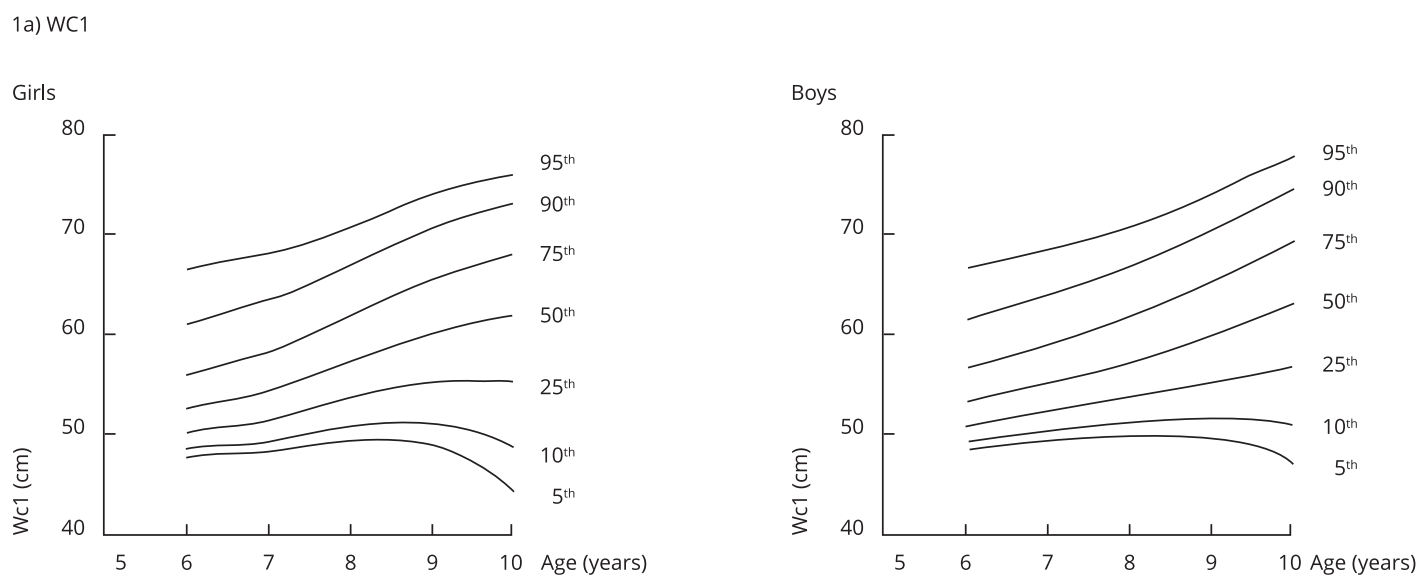

1b) WC2
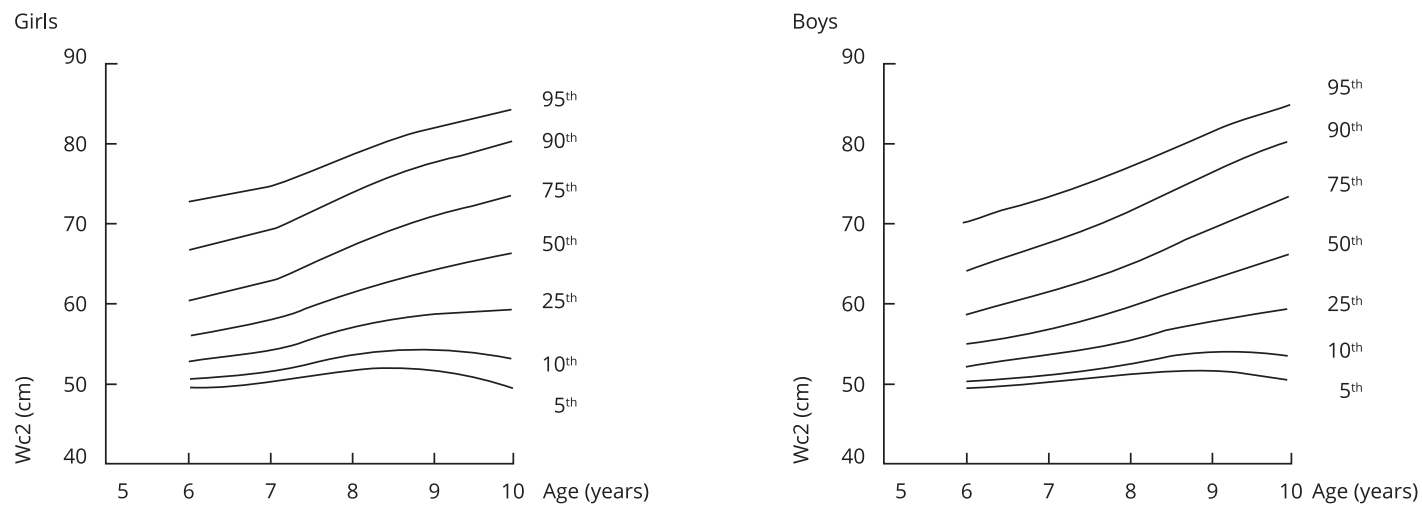

\section{Discussion}

Our findings showed that WC1 and WC2 are screening tools to predict MetS risk in different ages and both sexes. WC1 and WC2 were effective in predicting low HDL-c, high blood pressure and hypertriglyceridemia in girls. Cardiovascular risk factors predicted by WC1 and WC2, in boys, were high blood pressure and hypertriglyceridemia, at some ages. To our knowledge, there are no published studies on WC percentile curves as screening tools to predict cardiovascular risk factors and MetS risk for Brazilian children. The goal of this study was to develop reference WC values for Brazilian children aged between 6-10 years old, and to evaluate WC performance in predicting MetS risk. WC1 and WC2 were good MetS risk predictors for both sexes and in different ages, the AUC was significant (AUC $>0.79$ and $\mathrm{p}<0.05$ ) and the sensitivity values (from $71.4 \%$ to $100 \%$ ) were considered suitable screening tools 29 . We showed that WC cutoff points have potential to detect children with MetS risk and that these reference values could be used in clinical practice. 
Table 2

Smoothed age-specific percentile values for waist circumference measurement at the narrowest point (WC1) and at the umbilicus level (WC2) in 6-10-year-old boys and girls. Uberaba, Minas Gerais State, Brazil, 2011-2012.

\begin{tabular}{|c|c|c|c|c|c|c|c|c|c|c|}
\hline Age (years) & $\mathbf{L}$ & M & $\mathbf{S}$ & $5^{\text {th }}$ & 10th & 25th & 50 th & 75th & 90th & 95th \\
\hline & \multicolumn{10}{|c|}{ WC1 percentiles $(\mathrm{cm})$} \\
\hline \multicolumn{11}{|l|}{ Girls } \\
\hline 6 & -5.73 & 52.44 & 0.08 & 47.59 & 48.42 & 50.06 & 52.44 & 55.87 & 60.98 & 66.46 \\
\hline 7 & -3.86 & 54.18 & 0.09 & 48.11 & 49.17 & 51.25 & 54.18 & 58.15 & 63.36 & 67.92 \\
\hline 8 & -1.98 & 57.18 & 0.11 & 49.26 & 50.73 & 53.50 & 57.18 & 61.74 & 66.92 & 70.71 \\
\hline 9 & -0.07 & 60.05 & 0.13 & 48.89 & 51.14 & 55.17 & 60.05 & 65.39 & 70.63 & 73.99 \\
\hline 10 & 1.85 & 61.85 & 0.15 & 44.16 & 48.54 & 55.18 & 61.85 & 67.96 & 73.08 & 76.00 \\
\hline \multicolumn{11}{|l|}{ Boys } \\
\hline 6 & -5.77 & 53.36 & 0.08 & 48.54 & 49.37 & 51.00 & 53.36 & 56.73 & 61.66 & 66.79 \\
\hline 7 & -4.03 & 55.27 & 0.09 & 49.33 & 50.38 & 52.42 & 55.27 & 59.13 & 64.18 & 68.58 \\
\hline 8 & -2.27 & 57.40 & 0.10 & 49.82 & 51.21 & 53.86 & 57.40 & 61.84 & 66.99 & 70.84 \\
\hline 9 & -0.49 & 60.11 & 0.12 & 49.66 & 51.72 & 55.46 & 60.11 & 65.37 & 70.70 & 74.21 \\
\hline 10 & 1.33 & 63.25 & 0.15 & 47.14 & 50.84 & 56.83 & 63.25 & 69.46 & 74.91 & 78.10 \\
\hline & \multicolumn{10}{|c|}{ WC2 Percentiles $(\mathrm{cm})$} \\
\hline \multicolumn{11}{|l|}{ Girls } \\
\hline 6 & -3.87 & 56.05 & 0.10 & 49.45 & 50.59 & 52.84 & 56.05 & 60.51 & 66.61 & 72.23 \\
\hline 7 & -2.86 & 57.91 & 0.11 & 50.09 & 51.48 & 54.16 & 57.91 & 62.90 & 69.24 & 74.51 \\
\hline 8 & -1.85 & 61.37 & 0.12 & 51.77 & 53.52 & 56.86 & 61.37 & 67.07 & 73.74 & 78.76 \\
\hline 9 & -0.84 & 64.02 & 0.14 & 51.99 & 54.27 & 58.52 & 64.02 & 70.55 & 77.57 & 82.40 \\
\hline 10 & 0.17 & 65.86 & 0.16 & 50.46 & 53.58 & 59.13 & 65.86 & 73.20 & 80.37 & 84.93 \\
\hline \multicolumn{11}{|l|}{ Boys } \\
\hline 6 & -5.39 & 55.02 & 0.08 & 49.68 & 50.59 & 52.40 & 55.02 & 58.80 & 64.41 & 70.36 \\
\hline 7 & -3.98 & 56.98 & 0.10 & 50.35 & 51.50 & 53.76 & 56.98 & 61.48 & 67.68 & 73.47 \\
\hline 8 & -2.56 & 59.75 & 0.11 & 51.28 & 52.79 & 55.71 & 59.75 & 65.08 & 71.71 & 77.09 \\
\hline 9 & -1.13 & 63.30 & 0.13 & 52.02 & 54.13 & 58.09 & 63.30 & 69.61 & 76.57 & 81.50 \\
\hline 10 & 0.31 & 66.37 & 0.16 & 50.65 & 53.88 & 59.57 & 66.37 & 73.69 & 80.73 & 85.15 \\
\hline
\end{tabular}

L: for skewness; M: for median; S: for coefficient of variation.

Childhood obesity is a global public health problem that affects children in underdeveloped and developing countries, and is increasing at a rapid rate, especially in developing economies 30,31. Currently, there is an ongoing debate about the anthropometric measurements that should be used to diagnose MetS in children 32,33,34. Thus, this study contributes to this discussion and presents two different WC measurements as strong MetS predictors. Our findings corroborate results that have shown that different WC anatomical points did not affect relationships between visceral adiposity and cardiovascular risk factors in children 35 .

For nine years, a cohort of Brazilian children and adolescents (13.2 \pm 2.2 years) was monitored and it was found that the WC1 measured in the first wave was positively correlated with MetS diagnosed seven years later, as well as with abdominal fat accumulation detected in the last wave ${ }^{36}$. These results reinforce our findings, from the reports that the anthropometric measure predicts MetS and emphasize that WC is a simple and practical measurement capable of accurately determining the evolution of this syndrome.

Among anthropometric measures, WC was found to be the most effective predictor for MetS risk in children of both sexes 36,37,38,39. Considering the different anatomical points analyzed in this study, WC1 had a greater area under the ROC curve for 8 years old girls (AUC $=0.951$ ), i.e., $65.8 \mathrm{~cm}$, and 10 years old boys (AUC $=0.986$ ), $79.3 \mathrm{~cm}$. For WC2, the highest AUC was for 8 years old girls $(\mathrm{AUC}=0.936)$ and 7 years old boys $(\mathrm{AUC}=0.983$ ), i.e., 69.3 and $78.8 \mathrm{~cm}$, respectively (Table 3$)$. The 
Table 3

Cutoff points for waist circumference (WC1 and WC2) as predictors of metabolic syndrome in 6-10-year-old children. Uberaba, Minas Gerais State, Brazil, 2011-2012.

\begin{tabular}{|c|c|c|c|c|c|c|c|c|}
\hline Age (years) & Cutoff $(\mathrm{cm})$ & SE & SP & AUC & p-value & \multicolumn{2}{|c|}{$95 \% \mathrm{Cl}$} & Percentile \\
\hline & & & & & & & \multicolumn{2}{|c|}{ Girls } \\
\hline \multicolumn{9}{|l|}{ WC1 } \\
\hline 6 & - & - & - & 0.732 & 0.265 & 0.428 & 1.000 & - \\
\hline 7 & 65.8 & 75.0 & 92.1 & 0.908 & 0.006 & 0.798 & 1.000 & $>90$ \\
\hline 8 & 65.8 & 100.0 & 87.5 & 0.951 & 0.008 & 0.885 & 1.000 & $>75$ \\
\hline 9 & 61.1 & 85.7 & 66.7 & 0.868 & 0.001 & 0.704 & 1.000 & $>50$ \\
\hline 10 & 73.5 & 100.0 & 90.8 & 0.931 & 0.001 & 0.874 & 0.988 & $>75$ \\
\hline \multicolumn{9}{|l|}{ WC2 } \\
\hline 6 & - & - & - & 0.715 & 0.303 & 0.406 & 1.000 & - \\
\hline 7 & 68.8 & 75.0 & 89.0 & 0.891 & 0.008 & 0.770 & 1.000 & $>75$ \\
\hline 8 & 69.3 & 100.0 & 82.1 & 0.936 & 0.010 & 0.853 & 1.000 & $>75$ \\
\hline 9 & 65.2 & 100.0 & 60.4 & 0.930 & 0.000 & 0.827 & 1.000 & $>50$ \\
\hline 10 & 78.5 & 100.0 & 88.5 & 0.934 & 0.001 & 0.879 & 0.990 & $>75$ \\
\hline \multicolumn{9}{|l|}{ Boys } \\
\hline \multicolumn{9}{|l|}{ WC1 } \\
\hline 6 & - & - & - & 0.973 & 0.106 & 0.935 & 1.000 & - \\
\hline 7 & 74.1 & 100.0 & 97.8 & 0.980 & 0.021 & 0.952 & 1.000 & $>95$ \\
\hline 8 * & - & - & - & - & - & - & - & - \\
\hline 9 & 77.5 & 100.0 & 95.5 & 0.971 & 0.002 & 0.936 & 1.000 & $>95$ \\
\hline 10 & 79.3 & 100 & 95.9 & 0.986 & 0.004 & 0.960 & 1.000 & $>95$ \\
\hline \multicolumn{9}{|l|}{ WC2 } \\
\hline 6 & - & - & - & 1.000 & 0.087 & 1.000 & 1.000 & - \\
\hline 7 & 78.8 & 100.0 & 97.8 & 0.983 & 0.020 & 0.956 & 1.000 & $>95$ \\
\hline 8 * & - & - & - & - & - & - & - & - \\
\hline 9 & 77.6 & 100.0 & 91.0 & 0.980 & 0.001 & 0.952 & 1.000 & $>95$ \\
\hline 10 & 79.3 & 100.0 & 87.8 & 0.977 & 0.005 & 0.944 & 1.000 & $>75$ \\
\hline
\end{tabular}

95\%Cl: 95\% confidence interval; SE: sensitivity; SP: specificity; AUC: area under the ROC curve; WC1: waist circumference measurement at the narrowest point; WC2: waist circumference measurement at the umbilicus level.

* ROC curve was not performed since there were no cases of metabolic syndrome.

best diagnostic values are those closest to an AUC of 1.00. Additionally, the risk of developing a set of cardiovascular risk factors was identified in the sample from the $50^{\text {th }}$ to $95^{\text {th }}$ percentiles. When observed by sex, girls were at risk for MetS at lower percentiles than boys ( $50^{\text {th }}-75^{\text {th }}$ vs. $7^{\text {th }}-95^{\text {th }}$ ).

We also provide some evidence regarding WC and cardiovascular risk factors in this age range. Sardinha et al. 40 found that anthropometric variables such as BMI, WC, and waist-to-height ratio were similar in discriminating children and adolescents at increased cardiometabolic risk, with areas under the ROC curve between 0.70 and 0.74 and the sensitivity (boys: $80.5-86.4 \%$; girls: 76.6-82.3\%) was markedly higher than specificity (boys: 51.85-59.4\%; girls: 60.8\%). Furthermore, comparatively, WC was more consistent and a better predictor of cardiovascular risk factors than waist-to-height ratio and BMI in Chinese children 41 . The study provided optimal age-and-sex-specific thresholds to identify individual and clustering risk factors and the thresholds for WC were $57.4-80.4 \mathrm{~cm}$ and 55.8 $69.6 \mathrm{~cm}$ in boys and girls, respectively. Researching this topic is important since a consensus among cut-off points is needed to support actions to prevent cardiovascular and metabolic problems early in life.

A study with Brazilian children ${ }^{1}$ pointed WC1 as a more accurate predictor of cardiovascular risk factors when compared to WC2 and suggested WC1 cutoff points for predicting MetS risk in 
Table 4

Cutoff points for waist circumference (WC1 and WC2) as predictors of cardiovascular risk factors in 6-10-year-old boys and girls. Uberaba, Minas Gerais State, Brazil, 2011-2012.

\begin{tabular}{|c|c|c|c|c|c|c|c|c|}
\hline Age (years) & AUC & p-value & \multicolumn{2}{|c|}{$95 \% \mathrm{Cl}$} & Cutoff $(\mathrm{cm})$ & SE & SP & Percentile \\
\hline \multicolumn{9}{|l|}{ Low HDL-c } \\
\hline \multicolumn{9}{|l|}{ WC1 } \\
\hline 6 & 0.475 & 0.708 & 0.341 & 0.608 & - & - & - & - \\
\hline 7 & 0.699 & 0.000 & 0.598 & 0.799 & 54.50 & 71.7 & 65.2 & $>50$ \\
\hline 8 & 0.607 & 0.046 & 0.503 & 0.712 & 56.5 & 62.7 & 55.9 & $<50$ \\
\hline 9 & 0.527 & 0.626 & 0.418 & 0.636 & - & - & - & - \\
\hline 10 & 0.527 & 0.626 & 0.418 & 0.636 & - & - & - & - \\
\hline \multicolumn{9}{|l|}{ WC2 } \\
\hline 6 & 0.484 & 0.814 & 0.349 & 0.619 & - & - & - & - \\
\hline 7 & 0.686 & 0.001 & 0.582 & 0.790 & 58.20 & 71.7 & 62.1 & $>50$ \\
\hline 8 & 0.630 & 0.015 & 0.528 & 0.732 & 61.80 & 60.8 & 58.8 & $>50$ \\
\hline 9 & 0.545 & 0.413 & 0.437 & 0.653 & - & - & - & - \\
\hline 10 & 0.545 & 0.413 & 0.437 & 0.653 & - & - & - & - \\
\hline \multicolumn{9}{|c|}{ Hyperglycemia } \\
\hline \multicolumn{9}{|l|}{ WC1 } \\
\hline 6 * & - & - & - & - & - & - & - & - \\
\hline 7 * & - & - & - & - & - & - & - & - \\
\hline 8 * & - & - & - & - & - & - & - & - \\
\hline 9 & 0.061 & 0.132 & 0.017 & 0.105 & - & - & - & - \\
\hline 10 & 0.394 & 0.474 & 0.191 & 0.598 & - & - & - & - \\
\hline \multicolumn{9}{|l|}{ WC2 } \\
\hline 6 & - & - & - & - & - & - & - & - \\
\hline 7 & - & - & - & - & - & - & - & - \\
\hline 8 & - & - & - & - & - & - & - & - \\
\hline 9 & 0.044 & 0.117 & 0.006 & 0.081 & - & - & - & - \\
\hline 10 & 0.383 & 0.428 & 0.176 & 0.590 & - & - & - & - \\
\hline \multicolumn{9}{|c|}{ High blood pressure } \\
\hline \multicolumn{9}{|l|}{ WC1 } \\
\hline 6 * & 0.669 & 0.034 & 0.519 & 0.820 & 56.1 & 60.0 & 76.6 & $>75$ \\
\hline 7 * & 0.588 & 0.252 & 0.427 & 0.748 & - & - & - & - \\
\hline 8 * & 0.587 & 0.253 & 0.419 & 0.754 & - & - & - & - \\
\hline 9 & 0.654 & 0.012 & 0.521 & 0.787 & 57.1 & 74.1 & 40.0 & $>25$ \\
\hline 10 & 0.673 & 0.036 & 0.512 & 0.834 & 62.8 & 71.4 & 68.9 & $>50$ \\
\hline \multicolumn{9}{|l|}{ WC2 } \\
\hline 6 & 0.666 & 0.037 & 0.535 & 0.798 & 56.5 & 66.7 & 58.6 & $>25$ \\
\hline 7 & 0.569 & 0.370 & 0.404 & 0.733 & - & - & - & - \\
\hline 8 & 0.591 & 0.230 & 0.426 & 0.756 & - & - & - & - \\
\hline 9 & 0.686 & 0.003 & 0.558 & 0.814 & 63.8 & 66.7 & 53.6 & $>25$ \\
\hline 10 & 0.643 & 0.083 & 0.469 & 0.817 & 67.0 & 71.4 & 61.2 & $>50$ \\
\hline \multicolumn{9}{|c|}{ Hypertriglyceridemia } \\
\hline \multicolumn{9}{|l|}{ WC1 } \\
\hline 6 & 0.469 & 0.734 & 0.277 & 0.661 & - & - & - & - \\
\hline 7 & 0.762 & 0.002 & 0.615 & 0.908 & 57.1 & 78.6 & 76.5 & $>50$ \\
\hline 8 & 0.687 & 0.012 & 0.518 & 0.855 & 58.0 & 72.2 & 66.7 & $>50$ \\
\hline 9 & 0.628 & 0.081 & 0.478 & 0.777 & - & - & - & - \\
\hline 10 & 0.705 & 0.000 & 0.594 & 0.816 & 62.8 & 67.6 & 69.0 & $>50$ \\
\hline
\end{tabular}

(continues) 
Table 4 (continued)

\begin{tabular}{|c|c|c|c|c|c|c|c|c|}
\hline Age (years) & AUC & p-value & \multicolumn{2}{|c|}{$95 \% \mathrm{Cl}$} & Cutoff $(\mathrm{cm})$ & SE & SP & Percentile \\
\hline \multicolumn{9}{|l|}{ Girls } \\
\hline \multicolumn{9}{|l|}{ WC2 } \\
\hline 6 & 0.477 & 0.798 & 0.292 & 0.661 & - & - & - & - \\
\hline 7 & 0.769 & 0.001 & 0.622 & 0.916 & 60.5 & 85.7 & 73.5 & $>50$ \\
\hline 8 & 0.687 & 0.011 & 0.534 & 0.840 & 64.2 & 72.2 & 72.5 & $>50$ \\
\hline 9 & 0.665 & 0.024 & 0.521 & 0.809 & 65.2 & 68.4 & 58.9 & $>50$ \\
\hline 10 & 0.695 & 0.001 & 0.585 & 0.805 & 67.0 & 67.6 & 62.1 & $>50$ \\
\hline \multicolumn{9}{|l|}{ Boys } \\
\hline \multicolumn{9}{|l|}{ Low HDL-C } \\
\hline \multicolumn{9}{|l|}{ WC1 } \\
\hline 6 & 0.527 & 0.626 & 0.418 & 0.636 & - & - & - & - \\
\hline 7 & 0.603 & 0.099 & 0.482 & 0.724 & - & - & - & - \\
\hline 8 & 0.480 & 0.754 & 0.350 & 0.610 & - & - & - & - \\
\hline 9 & 0.480 & 0.754 & 0.350 & 0.610 & - & - & - & - \\
\hline 10 & 0.668 & 0.005 & 0.556 & 0.780 & 61.6 & 60.5 & 60.7 & $>25$ \\
\hline \multicolumn{9}{|l|}{ WC2 } \\
\hline 6 & 0.545 & 0.413 & 0.437 & 0.653 & - & - & - & - \\
\hline 7 & 0.577 & 0.214 & 0.455 & 0.700 & - & - & - & - \\
\hline 8 & 0.457 & 0.501 & 0.328 & 0.587 & - & - & - & - \\
\hline 9 & 0.457 & 0.501 & 0.328 & 0.587 & - & - & - & - \\
\hline 10 & 0.642 & 0.028 & 0.517 & 0.745 & 65.5 & 60.5 & 59.7 & $>50$ \\
\hline \multicolumn{9}{|c|}{ Hyperglycemia } \\
\hline \multicolumn{9}{|l|}{ WC1 } \\
\hline 6 & 0.405 & 0.745 & 0.296 & 0.514 & - & - & - & - \\
\hline 7 & 0.985 & 0.097 & 0.959 & 1.000 & - & - & - & - \\
\hline 8 & 0.859 & 0.219 & 0.788 & 0.930 & - & - & - & - \\
\hline 9 & 0.066 & 0.136 & 0.017 & 0.115 & - & - & - & - \\
\hline 10 & 0.899 & 0.171 & 0.840 & 0.958 & - & - & - & - \\
\hline \multicolumn{9}{|l|}{ WC2 } \\
\hline 6 & 0.468 & 0.914 & 0.357 & 0.579 & - & - & - & - \\
\hline 7 & 0.990 & 0.093 & 0.970 & 1.000 & - & - & - & - \\
\hline 8 & 0.913 & 0.157 & 0.855 & 0.971 & - & - & - & - \\
\hline 9 & 0.086 & 0.156 & 0.028 & 0.144 & - & - & - & - \\
\hline 10 & 0.889 & 0.182 & 0.827 & 0.951 & - & - & - & - \\
\hline \multicolumn{9}{|c|}{ High blood pressure } \\
\hline \multicolumn{9}{|l|}{ WC1 } \\
\hline 6 & 0.505 & 0.957 & 0.339 & 0.670 & - & - & - & - \\
\hline 7 & 0.746 & 0.009 & 0.572 & 0.920 & 56.4 & 70.0 & 59.3 & $>50$ \\
\hline 8 & 0.522 & 0.774 & 0.354 & 0.690 & - & - & - & - \\
\hline 9 & 0.809 & 0.000 & 0.708 & 0.909 & 60.8 & 85.7 & 73.7 & $>50$ \\
\hline 10 & 0.800 & 0.000 & 0.653 & 0.947 & 65.8 & 80.0 & 74.3 & $>50$ \\
\hline \multicolumn{9}{|l|}{ WC2 } \\
\hline 6 & 0.555 & 0.518 & 0.391 & 0.719 & - & - & - & - \\
\hline 7 & 0.774 & 0.004 & 0.615 & 0.933 & 62.3 & 70.0 & 80.0 & $>75$ \\
\hline 8 & 0.517 & 0.822 & 0.348 & 0.687 & - & - & - & - \\
\hline 9 & 0.796 & 0.000 & 0.696 & 0.897 & 65.8 & 78.6 & 70.4 & $>50$ \\
\hline 10 & 0.773 & 0.001 & 0.613 & 0.934 & 67.8 & 80.0 & 65.7 & $>50$ \\
\hline
\end{tabular}

(continues) 
Table 4 (continued)

\begin{tabular}{|c|c|c|c|c|c|c|c|c|}
\hline Age (years) & AUC & p-value & \multicolumn{2}{|c|}{$95 \% \mathrm{Cl}$} & Cutoff $(\mathrm{cm})$ & SE & SP & Percentile \\
\hline \multicolumn{9}{|l|}{ Boys } \\
\hline \multicolumn{9}{|c|}{ Hypertriglyceridemia } \\
\hline \multicolumn{9}{|l|}{ WC1 } \\
\hline 6 & 0.499 & 0.992 & 0.159 & 0.838 & - & - & - & - \\
\hline 7 & 0.657 & 0.105 & 0.465 & 0.849 & - & - & - & - \\
\hline 8 & 0.519 & 0.851 & 0.321 & 0.717 & - & - & - & - \\
\hline 9 & 0.823 & 0.000 & 0.725 & 0.921 & 61.1 & 78.6 & 79.1 & $>50$ \\
\hline 10 & 0.727 & 0.004 & 0.582 & 0.873 & 65.8 & 68.8 & 72.1 & $>50$ \\
\hline \multicolumn{9}{|l|}{ WC2 } \\
\hline 6 & 0.497 & 0.984 & 0.191 & 0.804 & - & - & - & - \\
\hline 7 & 0.669 & 0.080 & 0.485 & 0.854 & - & - & - & - \\
\hline 8 & 0.619 & 0.242 & 0.446 & 0.792 & - & - & - & - \\
\hline 9 & 0.849 & 0.000 & 0.763 & 0.934 & 64.8 & 92.9 & 68.6 & $>50$ \\
\hline 10 & 0.747 & 0.002 & 0.622 & 0.872 & 65.8 & 75.0 & 59.3 & $>25$ \\
\hline
\end{tabular}

95\%Cl: 95\% confidence interval; SE: sensitivity; SP: specificity; AUC: area under the ROC curve; WC1: waist circumference measurement at the narrowest point; WC2: waist circumference measurement at the umbilicus level.

* ROC curve was not performed since there were no cases of hyperglycemia.

Brazilian girls and boys $(9.9 \pm 0.7$ years $)$, WC1 $>61.3 \mathrm{~cm}(67$ th percentile) and WC1 $>66 \mathrm{~cm}(79$ th percentile), respectively. Lunardi et al. 42 suggested cutoff points of WC1 $>65.7 \mathrm{~cm}$ (69th percentile) to predict total cholesterol alteration, WC1 $>65.7 \mathrm{~cm}$ to predict LDL-c alteration in Brazilian boys (11 years) and WC1 $>65.1 \mathrm{~cm}$ to predict hypertriglyceridemia risk in girls ( 77 th percentile). Published data 1,42 shows WC1 values very close to the cutoff points suggested for WC1 (10 year-old children) in this study (Table 3).

WC2 was significantly associated with the MetS component in Argentinean children aged $9.2 \pm$ 2.2 years 43 . The authors highlighted that children with abdominal obesity ( $>90^{\text {th }}$ percentile of the sample) had an increased risk of cardiovascular risk factors such as hypertension, dyslipidemia and insulin resistance. Two years later, the same research group 39 tested the WC2 accuracy in predicting MetS risk in Argentinean school children aged $8.7 \pm 2.4$ years, and pointed the WC2 $>71.3^{\text {th }}$ percentile $(\mathrm{SE}=58.9 \%$ and $\mathrm{SP}=63.1 \%)$ as a predictor of MetS risk. The authors suggested $\mathrm{WC} 2>75^{\text {th }}$ percentile as an important tool in the prediction of type 2 diabetes and cardiovascular risk factors in children. Koulouridis et al. 44 showed WC2 $>90^{\text {th }}$ percentile as one of the diagnostic criteria of MetS, suggesting $64.0 \mathrm{~cm}$ and $64.5 \mathrm{~cm}$ as the cutoff points for Greek boys and girls under the age of 11 years, respectively. We can observe that the differences in cutoff points and cardiovascular risk factors used in these studies may be the key to the differences found. Caution must be exercised for comparisons, despite these results always showing an alarming picture in children health.

Ferranti et al. 45 defined a waist circumference cutoff point for MetS diagnosis around the $75^{\text {th }}$ percentile. In this study, the WC1 cutoff points suggested to predict MetS risk were distributed between the 50th (9-10 years.) and 75th (6-8 years.) percentiles. The WC2 cutoff points were situated solely above the 50th percentile for girls in all ages and for boys, between the 75th (6-7 years) and 50th (8-10 years) percentiles. WC2 showed lower percentile values in predicting MetS risk, which was confirmed by the ANOVA test, finding significant differences between the anatomical point with higher WC2 values in relation to WC1.

WC values increased with age in both sexes, regardless of the anatomical point, which corroborates with the current studies 12,13,46. Values above the 50th percentile were chosen as a cutoff point for both WC types assessed to predict cardiovascular risk factors and MetS risk in children. The choice for the cutoff point should be made when there is greater balance between sensitivity and specificity values, cutoff points with higher sensitivity should be preferred when intending a screening, since most children with MetS will be included, despite the increased number of false positives. 
Studies performed in countries, like Portugal 47, Japan 48, Kuwait 49 and Turkey 50, have descriptive percentile curves without cardiovascular risk factors prediction. This cross-sectional study was the first to show WC values to predict cardiovascular risk factors and MetS risk in Brazilian children, presenting age-and-sex-specific smoothed curves for the selected percentile references for the WC of 6-10 years old Brazilian children. We showed a draw of two reference values, which added Brazilian children to the global WC database, thus, allowing our data to be compared with other WC reference curves around the world. Furthermore, we strongly recommend the use of WC1 reference values in clinical practice for Brazilian children, as better AUC values were found when compared with WC2.

Regarding this potential, these results are applicable as a simple screening tool. We believe that our data can assist MetS risk screening in Brazilian children, supporting health promotion strategies and health professionals from different expertise areas, especially those who work directly with this population. Future studies must investigate factors associated with increased WC in children, as well as approaches focusing on lifestyle changes, especially in children diagnosed with MetS risk.

This study has some limitations. The data are representative for a Southeastern Brazilian city, but not for the country. There is no consensus regarding MetS diagnosis in children under 10 years old, and we must be cautious when using an adapted MetS criteria. Other studies use different MetS diagnostic criteria and cutoff points for cardiovascular risk factors, which makes their data only partially comparable to ours. However, what must be emphasized is that regardless of the criterion used, waist circumference is strongly correlated to MetS and cardiovascular risk factors in children under 10 years old.

This study presented percentile curves for WC at two anatomical points in a representative children sample of a Southeastern Brazilian city. Furthermore, WC was shown to be a strong predictor of MetS and cardiovascular risk factors in children. These results can be applied in public health interventions seeking an early diagnosis of cardiovascular risk factors in 6-10-year-old Brazilian children.

\section{Contributors}

A. C. R. Andaki conceived the manuscript, performed the statistical analyses, interpreted the data and drafted the manuscript. E. L. Mendes partly contributed to the conception and design of the study, provided statistical expertise and critical revision. A. Santos interpreted the data and drafted the manuscript discussion. C. J. Brito interpreted the data and drafted the manuscript. A. L. A. Tinoco contributed to the analyses and interpretation of the data and provided critical revision of the manuscript. J. Mota provided critical revision of the manuscript. All authors provided critical feedback on the drafts, read and approved the final manuscript.

\section{Acknowledgments}

This study was funded by FAPEMIG (Minas Gerais State Research Foundation - CDS - APQ-0227911). The authors A. C. R. A., A. S. and C. J. B. received research funding from the Brazilian National Research Council, Brazilian Government Scholarship Program Science without Borders. The authors declare no conflict of interest. 


\section{References}

1. Andaki AC, Tinoco AL, Mendes EL, Andaki Junior R, Hills AP, Amorim PR. Different waist circumference measurements and prediction of cardiovascular risk factors and metabolic syndrome in children. Obes Res Clin Pract 2012; 6:e91-e174.

2. Andaki AC, Tinoco AL, Mendes EL, Andaki Junior R, Hills AP, Amorim PR. Anthropometry and physical activity level in the prediction of metabolic syndrome in children. Public Health Nutr 2014; 17:2287-94.

3. Savva SC, Tornaritis M, Savva ME, Kourides Y, Panagi A, Silikiotou N, et al. Waist circumference and waist-to-height ratio are better predictors of cardiovascular disease risk factors in children than body mass index. Int J Obes Relat Metab Disord 2000; 24:1453-8.

4. Khadilkar A, Ekbote V, Chiplonkar S, Khadilkar V, Kajale N, Kulkarni S, et al. Waist circumference percentiles in 2-18 year old Indian children. J Pediatr 2014; 164:1358-62 e2.

5. Katzmarzyk PT, Bouchard C. Where is the beef? Waist circumference is more highly correlated with BMI and total body fat than with abdominal visceral fat in children. Int J Obes (Lond) 2014; 8:753-4.

6. Sijtsma A, Bocca G, L'Abee C, Liem ET, Sauer PJ, Corpeleijn E. Waist-to-height ratio, waist circumference and BMI as indicators of percentage fat mass and cardiometabolic risk factors in children aged 3-7 years. Clin Nutr 2014; 33:311-5

7. Melo X, Santa-Clara H, Pimenta NM, Martins SS, Minderico CS, Fernhall B, et al. Intimamedia thickness in 11- to 13-year-old children: variation attributed to sedentary behavior, physical activity, cardiorespiratory fitness, and waist circumference. J Phys Act Health 2015; 12:610-7.

8. Walker JL, Murray TD, Eldridge J, Squires WG, Silvius P, Silvius E. The association between waist circumference and FITNESSGRAM(R) aerobic capacity classification in sixth-grade children. Pediatr Exerc Sci 2015; 27:488-93.

9. Gomez-Arbelaez D, Camacho PA, Cohen DD, Rincon-Romero K, Alvarado-Jurado L, Pinzon $S$, et al. Higher household income and the availability of electronic devices and transport at home are associated with higher waist circumference in Colombian children: the ACFIES study. Int J Environ Res Public Health 2014; 11:1834-43.

10. Schroder H, Ribas L, Koebnick C, Funtikova A, Gomez SF, Fito M, et al. Prevalence of abdominal obesity in Spanish children and adolescents. Do we need waist circumference measurements in pediatric practice? PLoS One 2014; 9:e87549.

11. Katzmarzyk PT. Waist circumference percentiles for Canadian youth 11-18y of age. Eur J Clin Nutr 2004; 58:1011-5.
12. Chaves R, Baxter-Jones A, Souza M, Santos D, Maia J. Height, weight, body composition, and waist circumference references for 7 - to 17 -year-old children from rural Portugal. Homo 2015 ; 66:264-77.

13. Anzo M, Inokuchi M, Matsuo N, Takayama JI, Hasegawa T. Waist circumference centiles by age and sex for Japanese children based on the 1978-1981 cross-sectional national survey data. Ann Hum Biol 2015; 42:56-61.

14. Eisenmann JC. Waist circumference percentiles for 7- to 15-year-old Australian children. Acta Paediatr 2005; 94:1182-5.

15. Matsushita R, Isojima T, Takaya R, Satake E, Yamaguchi R, Kitsuda K, et al. Development of waist circumference percentiles for Japanese children and an examination of their screening utility for childhood metabolic syndrome: a population-based cross-sectional study. BMC Public Health 2015; 15:1121.

16. Rerksuppaphol S, Rerksuppaphol L. Waist circumference, waist-to-height ratio and body mass index of Thai children: secular changes and updated reference standards. J Clin Diagn Res 2014; 8:PC05-9.

17. Okosun IS, Boltri JM, Eriksen MP, Hepburn VA. Trends in abdominal obesity in young people: United States 1988-2002. Ethn Dis 2006; 16:338-44.

18. Garnett SP, Baur LA, Cowell CT. The prevalence of increased central adiposity in Australian school children 1985 to 2007. Obes Rev 2011; 12:887-96.

19. Liu W, Lin R, Liu A, Du L, Chen Q. Prevalence and association between obesity and metabolic syndrome among Chinese elementary school children: a school-based survey. BMC Public Health 2010; 10:780.

20. Cole TJ, Bellizzi MC, Flegal KM, Dietz WH. Establishing a standard definition for child overweight and obesity worldwide: international survey. BMJ 2000; 320:1240-3.

21. Lohman TG, Roche AF, Martorell R. Anthropometric standardization reference manual. Champaign: Human Kinetics; 1988.

22. American Diabetes Association. (2) Classification and diagnosis of diabetes. Diabetes Care 2015; 38 Suppl 1:S8-16.

23. Expert Panel on Integrated Guidelines for Cardiovascular Health and Risk Reduction in Children and Adolescents; National Heart, Lung, and Blood Institute. Expert panel on integrated guidelines for cardiovascular health and risk reduction in children and adolescents: summary report. Pediatrics 2011; 128 Suppl 5:S21356.

24. Sociedade Brasileira de Cardiologia; Sociedade Brasileira de Hipertensão; Sociedade Brasileira de Nefrologia. VI Diretrizes Brasileiras de Hipertensão. Arq Bras Cardiol 2010; 95(1 Suppl):1-51. 
25. National High Blood Pressure Education Program Working Group on High Blood Pressure in Children and Adolescents. The fourth report on the diagnosis, evaluation, and treatment of high blood pressure in children and adolescents. Pediatrics 2004; 114(2 Suppl 4th Report):555-76.

26. Cole TJ, Green PJ. Smoothing reference centile curves: the LMS method and penalized likelihood. Stat Med 1992; 11:1305-19.

27. Cole TJ, Freeman JV, Preece MA. British 1990 growth reference centiles for weight, height, body mass index and head circumference fitted by maximum penalized likelihood. Stat Med 1998; 17:407-29.

28. Schisterman EF, Faraggi D, Reiser B, Trevisan M. Statistical inference for the area under the receiver operating characteristic curve in the presence of random measurement error. Am J Epidemiol 2001; 154:174-9.

29. Menezes AMB, Santos IS. Curso de epidemiologia básica para pneumologistas. 4a parte - epidemiologia clínica. J Pneumologia 1999; 25:321-6

30. Sharma VK, Joshi S, Joshi A, Kumar G, Arora H, Garg A. Does intravenous sildenafil clinically ameliorate pulmonary hypertension during perioperative management of congenital heart diseases in children? A prospective randomized study. Ann Card Anaesth 2015; 18:510-6.

31. Genovesi S, Antolini L, Giussani M. Hypertension screening in children: is it necessary? J Hypertens 2015; 33:2179-80.

32. Andaki ACR, Tinoco ALA, Mendes EL, Amorim PRS. Diagnostics criteria of metabolic syndrome in children. In: Garcia CML, Gonzalez PAP, editors. Metabolic syndrome: classification, risk factors and health impact. New York: Nova Science Publishers; 2012. p. 305-17.

33. Pergher RN, Melo ME, Halpern A, Mancini MC. Is a diagnosis of metabolic syndrome applicable to children? J Pediatr (Rio J.) 2010; 86:101-8.

34. Sinaiko AR. Metabolic syndrome in children. J Pediatr (Rio J.) 2012; 88:286-8.

35. Harrington DM, Staiano AE, Broyles ST, Gupta AK, Katzmarzyk PT. Waist circumference measurement site does not affect relationships with visceral adiposity and cardiometabolic risk factors in children. Pediatr Obes 2013; 8:199-206.

36. Spolidoro JV, Pitrez Filho ML, Vargas LT, Santana JC, Pitrez E, Hauschild JA, et al. Waist circumference in children and adolescents correlate with metabolic syndrome and fat deposits in young adults. Clin Nutr 2013; 32:93-7.

37. Panagiotopoulos C, Ronsley R, Kuzeljevic B, Davidson J. Waist circumference is a sensitive screening tool for assessment of metabolic syndrome risk in children treated with secondgeneration antipsychotics. Can J Psychiatry 2012; 57:34-44.

38. Moreno LA, Pineda I, Rodriguez G, Fleta J, Sarria $\mathrm{A}$, Bueno $\mathrm{M}$. Waist circumference for the screening of the metabolic syndrome in children. Acta Paediatr 2002; 91:1307-12.
39. Hirschler V, Maccallini G, Calcagno M, Aranda C, Jadzinsky M. Waist circumference identifies primary school children with metabolic syndrome abnormalities. Diabetes Technol Ther 2007; 9:149-57.

40. Sardinha LB, Santos DA, Silva AM, Grøntved A, Andersen LB, Ekelund U. A comparison between BMI, waist circumference, and waist-toheight ratio for identifying cardio-metabolic risk in children and adolescents. PLoS One 2016; 11:e0149351.

41. Ma L, Cai L, Deng L, Zhu Y, Ma J, Jing J, et al. Waist circumference is better than other anthropometric indices for predicting cardiovascular disease risk factors in chinese children: a cross-sectional study in Guangzhou. J Atheroscler Thromb 2016; 23:320-9.

42. Lunardi CC, Petroski EL. Índice de massa corporal, circunferência da cintura e dobra cutânea triciptal na predição de alterações lipídicas em crianças com 11 anos de idade. Arq Bras Endocrinol Metabol 2008; 52:1009-14.

43. Hirschler V, Aranda C, Calcagno ML, Maccalini G, Jadzinsky M. Can waist circumference identify children with the metabolic syndrome? Arch Pediatr Adolesc Med 2005; 159:740-4.

44. Koulouridis E, Georgalidis K, Kostimpa I, Koulouridis I, Krokida A, Houliara D. Metabolic syndrome risk factors and estimated glomerular filtration rate among children and adolescents. Pediatr Nephrol 2010; 25:491-8.

45. Ferranti SD, Gauvreau K, Ludwig DS, Neufeld EJ, Newburger JW, Rifai N. Prevalence of the metabolic syndrome in American adolescents: findings from the Third National Health and Nutrition Examination Survey. Circulation 2004; 110:2494-7.

46. Bacopoulou F, Efthymiou V, Landis G, Rentoumis A, Chrousos GP. Waist circumference, waist-to-hip ratio and waist-to-height ratio reference percentiles for abdominal obesity among Greek adolescents. BMC Pediatr 2015; 15:50.

47. Samuels J, Bell C, Samuel J, Swinford R. Management of hypertension in children and adolescents. Curr Cardiol Rep 2015; 17:107.

48. Inokuchi M, Matsuo N, Anzo M, Takayama JI, Hasegawa T. Age-dependent percentile for waist circumference for Japanese children based on the 1992-1994 cross-sectional national survey data. Eur J Pediatr 2007; 166:655-61.

49. Jackson RT, Al Hamad N, Prakash P, Al Somaie M. Waist circumference percentiles for Kuwaiti children and adolescents. Public Health Nutr 2011; 14:70-6.

50. Hatipoglu N, Ozturk A, Mazicioglu MM, Kurtoglu S, Seyhan S, Lokoglu F. Waist circumference percentiles for 7- to 17-year-old Turkish children and adolescents. Eur J Pediatr 2008; 167:383-9. 


\section{Resumo}

O estudo teve como objetivos desenvolver valores de referência para perímetro de cintura $(P C)$ em crianças brasileiras entre 6 e 10 anos de idade e avaliar o desempenho da PC na predição de fatores de risco cardiovasculares e de sindrome metabólica (SM) em crianças. O estudo epidemiológico transversal de base populacional incluiu 1.397 crianças entre 6 e 10 anos de idade, através de amostragem probabilística em escolas públicas e privadas em Uberaba, Minas Gerais, Brasil. A PC era medida no ponto mais estreito da cintura (PC1) e ao nivel do umbigo (PC2). Foram coletadas amostras de sangue e medidas de pressão arterial para determinar o diagnóstico de SM. Os seguintes fatores mostram associação significativa com PC: idade $(p=0,001)$, ponto anatômico (PC1 vs. PC2, $p=0,001)$ e interação sexo/ponto anatômico ( $p=$ $0,016)$. O método LMS foi utilizado para desenhar as curvas suavizadas especificas para sexo e idade, dos percentis 5, 10, 25, 50, 75, 90 e 95. A PC mostrou-se acurada para a predição de $S M$, para todas as idades [área sob a curva ROC (AUC) >0,79e $p<0,05]$, independente de sexo. Conclusão: o estudo apresentou curvas de percentis de PC em dois pontos anatómicos em uma amostra representativa de crianças brasileiras. Além disso, a PC mostrou ser forte preditor de fatores de risco cardiovasculares e SM em crianças.

Antropometria; Curva ROC; Desenvolvimento Infantil

\section{Resumen}

Los objetivos de este estudio fueron desarrollar valores de referencia para la circunferencia de cintura (WC por sus siglas en inglés), en niños brasileños entre 6-10 años de edad, y evaluar los resultados de WC para predecir factores de riesgo cardiovasculares y síndrome metabólico (MetS) en niños. Se trata de un estudio epidemiológico transversal, basado en población, en el que participaron 1.397 niños, de 6 a 10 años de edad con muestreo probabilístico, procedentes de escuelas públicas y privadas en la ciudad de Uberaba, Minas Gerais, Brasil. WC fue medido en el punto de cintura más estrecho (WC1) y al nivel del ombligo (WC2). Las muestras de sangre y presión arterial se recogieron para determinar el diagnóstico de MetS. Habia un significativo efecto de la edad $(p=0.001)$, punto anatómico (WC1 vs. WC2, $p=0.001$ ) y punto de interacción sexo-anatómico $(p=0.016)$ para el WC. Sexo uniforme y edad específica $5^{\circ}, 10^{\circ}, 25^{\circ}$, $50^{\circ}, 75^{\circ}, 90^{\circ}$ y $95^{\circ}$ curvas de percentil de WC1 $y$ WC2 fueron diseñados mediante el método LMS. WC fue exacto para predecir MetS en todas las edades [área bajo la curva ROC $(A U C)>0.79 y \mathrm{p}$ $<0.05]$, independientemente del sexo. Conclusión: este estudio presentó un percentil de curvas para $W C$ en dos puntos anatómicos en una muestra representativa de niños brasileños. Asimismo, la WC se mostró como un fuerte predictor de factores de riesgo cardiovasculares y MetS en niños.

Antropometría; Curva ROC; Desarrollo Infantil
Submitted on 19/Jun/2017

Final version resubmitted on 06/Mar/2018

Approved on 06/Apr/2018 